OPEN ACCESS

Edited by:

James David McFadyen,

Baker Heart and Diabetes

Institute, Australia

Reviewed by:

Hirotsugu Tsuchimochi,

National Cerebral and Cardiovascular

Center, Japan

Mitchell Moon

Baker Heart and Diabetes Institute, Australia

*Correspondence:

Lining Arnold Ju

arnold.ju@sydney.edu.au

Specialty section:

This article was submitted to

Cardiovascular Biologics and

Regenerative Medicine,

a section of the journal

Frontiers in Cardiovascular Medicine

Received: 29 August 2021

Accepted: 15 October 2021

Published: 25 November 2021

Citation:

Zhang Y, Ramasundara SDZ,

Preketes-tardiani RE, Cheng V, Lu H

and Ju LA (2021) Emerging

Microfluidic Approaches for Platelet

Mechanobiology and Interplay With

Circulatory Systems.

Front. Cardiovasc. Med. 8:766513.

doi: $10.3389 /$ fcvm.2021.766513

\section{Emerging Microfluidic Approaches for Platelet Mechanobiology and Interplay With Circulatory Systems}

\author{
Yingqi Zhang 1,2,3, Savindi De Zoysa Ramasundara ${ }^{2,3,4}$, Renee Ellen Preketes-tardiani ${ }^{1,2,3}$, \\ Vivian Cheng ${ }^{1}$, Hongxu Lu ${ }^{1,5}$ and Lining Arnold Ju ${ }^{1,2,3 *}$ \\ ${ }^{1}$ School of Biomedical Engineering, Faculty of Engineering, The University of Sydney, Darlington, NSW, Australia, ${ }^{2}$ Charles \\ Perkins Centre, The University of Sydney, Camperdown, NSW, Australia, ${ }^{3}$ Heart Research Institute, Newtown, NSW, \\ Australia, ${ }^{4}$ School of Medicine, The University of Notre Dame Sydney, Darlinghurst, NSW, Australia, ${ }^{5}$ Faculty of Science, \\ Institute for Biomedical Materials and Devices, The University of Technology Sydney, Ultimo, NSW, Australia
}

Understanding how platelets can sense and respond to hemodynamic forces in disturbed blood flow and complexed vasculature is crucial to the development of more effective and safer antithrombotic therapeutics. By incorporating diverse structural and functional designs, microfluidic technologies have emerged to mimic microvascular anatomies and hemodynamic microenvironments, which open the floodgates for fascinating platelet mechanobiology investigations. The latest endothelialized microfluidics can even recapitulate the crosstalk between platelets and the circulatory system, including the vessel walls and plasma proteins such as von Willebrand factor. Hereby, we highlight these exciting microfluidic applications to platelet mechanobiology and platelet-circulatory system interplay as implicated in thrombosis. Last but not least, we discuss the need for microfluidic standardization and summarize the commercially available microfluidic platforms for researchers to obtain reproducible and consistent results in the field.

\footnotetext{
Keywords: microfluidic, platelet, mechanobiology, thrombosis, hemodynamic, endothelial cells, von Willebrand factor, COVID-19
}

\section{INTRODUCTION}

While three determinants for thrombosis-hypercoagulability, endothelial dysfunction, and hemodynamics - are outlined in Virchow's triad (1), platelets play an essential role in arterial thrombosis. Their thrombotic functions are governed by a range of biomechanical factors and the underlying mechanobiology is a rapidly evolving field and topical research area (2). Intriguingly, platelets respond to flow disturbance in complex vessel architectures with respect to bifurcation, branching, stenosis and aneurysm (3), which inevitably produce local shear gradients (4-6), vorticity $(7)$ and even turbulence $(4,8)$. The biomechanical platelet adhesion and aggregation are primarily mediated by its two mechanoreceptors-glycoprotein Ib (GPIb) and glycoprotein IIb/IIIa (GPIIb/IIIa or integrin $\left.\alpha_{\text {IIb }} \beta_{3}\right)(9,10)$. Furthermore, to facilitate thrombus stabilization, platelets generate contractile forces which are mediated by cytoskeletal components (actin, tubulin, Arp2/3) and motor proteins (myosin and dynein) in response to the mechanical microenvironment $(11,12)$. Meanwhile, platelets interact with red blood cells, neutrophils, plasma proteins [von Willebrand factor (VWF), fibrinogen], and the vessel wall (endothelial cells) to exert their hemostatic and thrombotic functions (13). Increasingly, microfluidic technologies have emerged as powerful and 
indispensable approaches to investigate the mechanosensitive behaviors of platelets $(2,12,14)$. Numerous endothelialized microfluidics have been invented to model the interplay between platelets, endothelium and VWF under physiologically relevant hemodynamic microenvironments $(6,15,16)$. In the context of platelet mechanobiology, we summarize these state-of-the-art microfluidic methodologies that have recently been invented in the field.

\section{MICROFLUIDIC APPROACHES FOR INVESTIGATING PLATELET MECHANOBIOLOGY AND PLATELET-CIRCULATORY SYSTEM INTERPLAY}

Single-cell biomechanical nanotools such as atomic force microscopy (17), optical tweezers (18) and micropipette based adhesion assays (19) significantly advanced our understanding of platelet mechanobiology (20). However, a major shortcoming of these in vitro techniques is their inability to recapitulate the physiologically relevant hemodynamics in the context of platelet adhesion and aggregation (21). To this end, animal models of thrombosis and intravital microscopies have become more popular and accessible approaches for examining thrombus initiation, progression and propagation in vivo (22). Nevertheless, multiple fundamental limitations still exist with the animal approaches $(23,24)$ : (i) inter-species variabilities which prevent complete recapitulation of human disease pathogenesis; (ii) physiological variations between individual animals; (iii) lengthy ethical approval processes.

Shear-based in vitro assays, such as parallel plate flow chambers (25), cone-and-plate viscometers (26) and thromboelastometers (27) have been widely used for investigations of human blood samples under physiologically relevant shear rates and shear stresses $(5,28-30)$. Although these methods were instrumental in the current understanding of shear dependent platelet thrombosis, to some extent, they are limited due to the need for large sample volumes, low throughput, and their inability to accurately emulate vascular architectures and mechanical properties of the circulatory system (31-33). To these points, microfluidic techniques have rapidly emerged as complementary humanized models for investigations of platelet mechanobiology and biomechanical thrombosis. More recently, the International Society of Thrombosis and Hemostasis (ISTH) have even made recommendations for producing reliable microfluidic devices with reproducible thrombogenic coating and consistent hemodynamic design (34). This represents a major step forward in promoting microfluidic technologies for future pre-clinical and diagnostic testing methods in hemostasis and thrombosis.

Moreover, while most of the research interests focus on large vessel thrombosis, there is an emerging trend toward understanding thrombosis in the microcirculation which is significantly implicated in a variety of systemic disorders such as: Thrombotic Thrombocytopenia Purpura (TTP) and
Hemolytic Uremic Syndrome (HUS) which lead to multiorgan dysfunction syndrome and death $(35,36)$; and localized organ injuries resulting from trauma, ischemia-reperfusion, transplant rejection, disseminated intravascular coagulation, sickle cell disease and more recently COVID-19 (35, 37, 38). The microfluidic approach can effectively recapitulate the anatomical structures within such small scale as opposed to conventional shear-based assays (39). The recent advancements of microfluidic designs and the micro and nano fabricationparticularly the shift from devices with a single layer to those with multiple-layered structures (40), and from parallel straight channels to complex geometries (15) - have enabled the investigation of platelet mechanobiology in more complicated vascular biomechanical microenvironments.

In the past decade, soft lithography has rapidly grown to enable high-fidelity micropatterning. Parallel straight channels can simply be fabricated by the single-layered photolithography and poly(dimethylsiloxane) (PDMS) casting processes, resulting in a vascular geometry with laminar flow (5, 16, 41, 42). When connected with external syringe pumps, such simple microfluidic setting enables systematic investigation of shear dependent platelet adhesion (43) and aggregation (44). Additionally, vascular cell cultures can be incorporated; specifically, endothelialized microchannels can model the interaction between blood components and the vessel wall under physiological hemodynamic parameters (45), or even when subjected to proinflammatory or other endothelial diseased conditions (16).

Furthermore, single-layered microfluidics can emulate more complex vessel structures, such as bifurcation (39), 2D stenosis (46), network (47), and micropost array (40) to recapitulate flow disturbance induced platelet activation, adhesion, contraction and aggregation $(48,49)$. The mechanical stimuli investigated include elevated shear stress $(50,51)$, shear rate gradient $(52$ $54)$, vorticity $(7)$ and turbulence $(8,55)$ at different locations of the microchannel. Moreover, lining the microchannel with endothelial cells enables the investigation of prothrombotic synergistic effects of hemodynamic forces, blood cells and the vessel wall $(56,57)$. Rapid prototyping methods such as vertical milling molding (58) and 3D printing (56) enable fabrication of microfluidics with circular structures and variations in the $\mathrm{z}$-direction. PDMS casting around poly(methyl methacrylate) (PMMA) optical fibers can also create circular channels (59, 60). As such, these recent microfluidic advancements provide great means for examining how shear stress, shear rate gradients, vorticity, blood viscosity and contractility affect platelet thrombotic functions.

In the past 3 years, in vitro lung (61), blood vessel (62), and tumor models (63) were made on microfluidics (64). These biomimetic organ-on-a-chip models recapitulate the structural, functional, and mechanical aspects of vascular microenvironments. Obviously, emulating such hierarchical organs requires emerging microfabrication methods including 3D bioprinting (65), double-layered soft lithography (66), injection molding (67), and micropattern stamping (68). This allows the production of multiple-layered structures via onetime or assembled fabrication. The composite microsystems 
can support tissue coculture and the channel-channel interface, thereby enabling studies of bidirectional tissue signaling across the endothelial barrier (61). More importantly, external mechanical manipulation apparatus (e.g., stiff ECM matrices, actuation chambers) can be integrated for disease-specific studies and rapid drug screening $(55,69)$. Although multiplelayered microfluidics incur higher fabrication requirements including precise alignment and assembly, they present physiologically relevant hemodynamic microenvironments for better biomimetic performance.

Last but not least, the transparency of PDMS material is greatly compatible with advanced microscopies to visualize thrombotic dynamics in real time. These PDMS or equivalent microfluidics thus offer a versatile way to evaluate platelet activation, adhesion, aggregation, morphological change, and soluble agonist secretion under hemodynamic control. A range of studies have delved into reproducing diverse vessel architectures to investigate how biomechanical factors (e.g., shear stress, shear gradients and traction forces) and the circulatory components (e.g., vessel endothelium and VWF) regulate platelet functions. As shown in the Figure 1 and Table 1, we summarize these recent lab-on-chip approaches in the context of platelet mechanobiology and thrombosis.

\section{Shear Dependent Platelet Adhesion}

Earlier endeavors in the field focused on developing highthroughput microfluidic systems with parallel straight microchannels to assess shear effects on platelet thrombotic functions (21). One of the novel straight channel microfluidic models was developed by Gutierrez et al., that validated the use of adhesion assays with VWF, fibrinogen, or collagen-coated substrates (66). The model contained eight rectilinear channels that covered a 100-fold range of shear rates to recapitulate the venous and arterial flow conditions. Such approaches can achieve high-throughput examination and avoid matrix variability between different microchannels. Similar studies were later conducted by Conant et al., who developed a $2 \mathrm{D}$ narrowing microfluidic device consisting of 24 microchannels coated with a range of matrix proteins on a 48 -well plate. Each of their microchannels was coupled with two wells (Figure 1A) (70). By changing the flow inputs with a syringe pump, platelet adhesion assays were conducted under controllable physiological and pathological hemodynamic conditions. These two highthroughput microfluidic systems substantially shortened the time for platelet adhesion analysis. As a key advantage, these microdevices use small volume of blood samples to produce a range of replicable results.

\section{Flow Disturbance Dependent Platelet Aggregation}

In pathological contexts, the aforementioned straight microfluidics could not effectively recapitulate flow disturbance at stenotic and bifurcated vasculatures (74).

To this end, Tovar-Lopez et al. proposed a $2 \mathrm{D}$ stenosed microfluidic model to assess the role of shear gradients on platelet aggregation at three observation zones: the pre-stenosis for shear acceleration; the apex of stenosis for maximum shear; and the post-stenosis for shear deceleration (46). Interestingly, stable platelet aggregation was only found at the post-stenotic zone with shear deceleration. Costa et al. presented similar observation in $3 \mathrm{D}$ coronary artery mimicking microfluidics. The $3 \mathrm{D}$ vessel geometries and stenoses were reconstructed from computed tomography angiography, and then manufactured into a microfluidic channel using stereolithography 3D-printing and PDMS soft lithography (56). The computational fluid dynamic simulation identified that backflow at the distal stenosis associates with rapid platelet aggregation. These results were comparable to the studies by Menon et al., using circular microfluidics with various degrees of stenosis in $2 \mathrm{D}$ and $3 \mathrm{D}$ (57). When Vascularized with endothelial cells, these microfluidics revealed that elevated shear stress at the stenotic regions activated endothelial cells into pro-thrombotic and proinflammatory states. Nevertheless, the round surface of these circular microfluidics makes it difficult in visualizing platelet adhesion and aggregation.

In addition, new microfluidic designs were made to investigate the prothrombotic role of shear gradients with respect to vessel bifurcations. The bifurcations produce flow separations, shear gradients, and oscillatory flows which promote atherosclerotic lesion initiation and thrombus formation (75). Li et al. designed a bifurcated stenosis device to mimic constricted regions in coronary arteries, where a mother microchannel is subdivided into two smaller daughter microchannels (73). Occlusive thrombi were formed extensively at higher shear rates between 1,500 and $4,000 \mathrm{~s}^{-1}$, but not at lower shear rates $<1,500 \mathrm{~s}^{-1}$. Additionally, the complex hemodynamics at vessel bifurcations were also investigated in a multi-bypass ladder model developed by Zilberman-Rudenko et al. which consisted of two parallel channels and 10 T-junction-like bypasses (47). Thrombus formation was found to be heightened at channel intersections which displaying skewed flow profiles, stagnation zones, flow separation, recirculation zones, and shear gradients.

Furthermore, research has been undertaken into automating the occlusion time calculation as thrombi form. Jain et al. designed a stenosed arteriole mimicking microfluidic device with multiple regions of pre- and post-stenosis (Figure 1C) (71). A mathematical model was used to quantitate shear gradient induced thrombosis in real time, indicating the steady reaction, growth, and saturation phases of thrombus development. They have also demonstrated the use of this microdevice to assess antithrombotic drug effects on individual patients.

\section{Platelet Contraction}

Activated platelets in newly formed aggregates are highly contractile with respect to thrombus stabilization and consolidation (76-81). Recent studies have sought to analyze the degree of forceful cytoskeletal contractions in activated platelets. Notably, Muthard and Diamond designed a microblock microfluidic model composed of a primary flow channel and a side scaffold channel to investigate platelet contractile forces during thrombus formation under arterial shear conditions (77). Platelet contraction occurred dramatically during flow arrest, and a consequent increase in the clot permeability was observed $(77,78)$. While Liang et al. quantified platelet retraction 
A

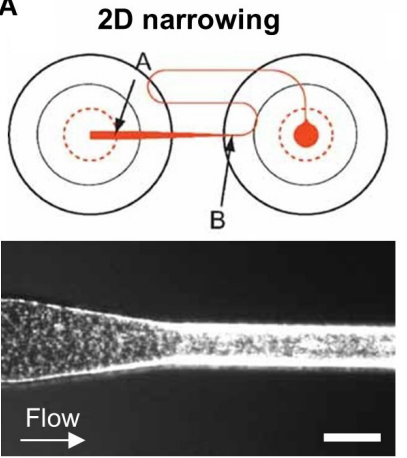

B 3D coronary artery-on-chip

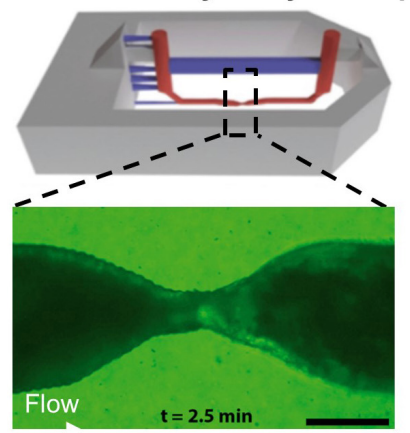

C

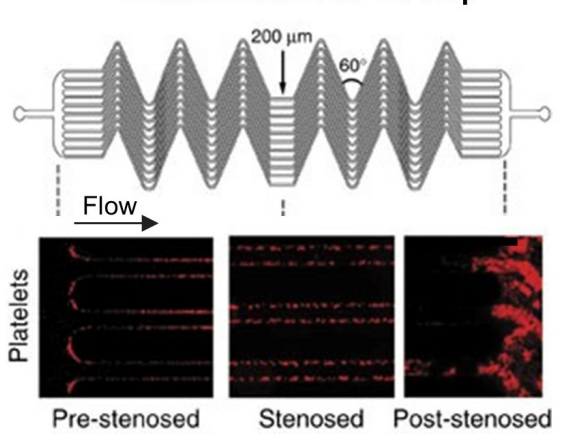

Stenosed arteriole-on-chip

Stenosed Post-stenosed
D

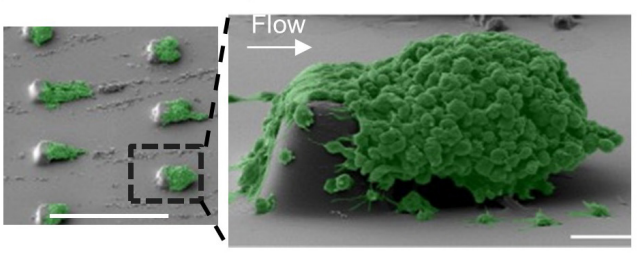

F 2D atherosclerotic vessel-on-chip
E

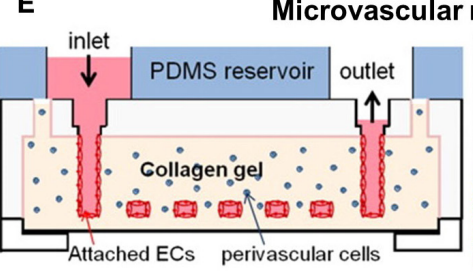

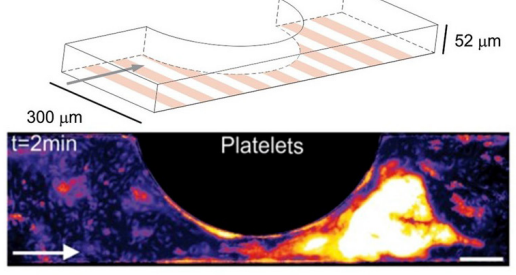

G

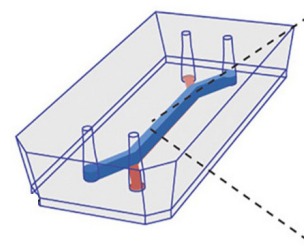

Triple-layered lung alveolus-on-chip

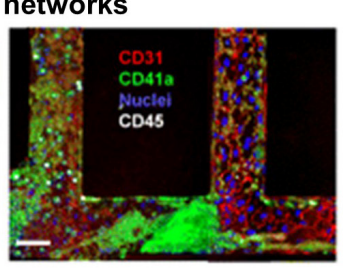

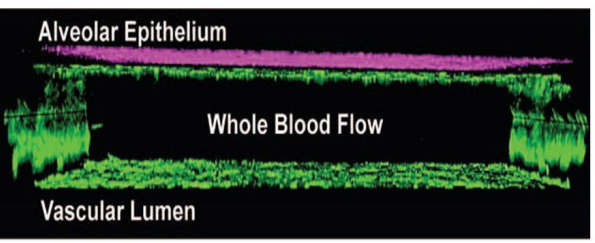

FIGURE 1 | Microfluidic models for the study of platelet mechanobiology and its interplay with circulatory system in thrombus development. (A) A 2D Narrowing channel from Conant et al. (70); Top: A well-plate microfluidic design, where arrow A indicates microfluidic channel entrance and arrow B indicates the neckdown region; Bottom: Platelet behavior at the neckdown zone. Scale bar $=100 \mu \mathrm{m}$. (B) A 3D coronary artery-on-chip model from Costa et al. (56); Top: Animation image of the 3D microfluidic model; Bottom: Fluorescence image of platelet aggregation at stenosis. Scale bar $=200 \mu \mathrm{m}$. (C) Stenosed arteriole mimicking microfluidic device from Jain et al. (71); A network of parallel stenosed microchannels which contain multiple regions of pre-stenosis, stenosis and post-stenosis. (D) Block-post contractile sensor from Ting et al. (40); SEM images of platelet aggregation on a micropost microfluidic device. Scale bar $=10 \mu \mathrm{m}$. (E) Microvascular networks from Zheng et al. (67); Left: Schematic of the multiple-layered collagen network in the microfluidic device; Right: Confocal image of the endothelialized microfluidic channel with platelet (green), leucocytes (white) adhesion and aggregation. Scale bar $=50 \mu \mathrm{m}$. (F) A 2D atherosclerotic vessel-on-chip model from Westein et al. (6); Left: Schematic diagram of microfluidic device incorporating microchannels with varying degrees of stenosis (20-80\%); Right: Confocal images of platelet aggregates at the endothelialized stenotic region upon blood perfusion. Scale bar $=100 \mu \mathrm{m}$. (G) Triple-layered lung alveolus-on-chip model from Jain et al. (61); Left: Schematic of the three-layered alveolus-on-a-chip model; Right: Confocal image of endothelial and epithelial cell coculture in the device.

force without physiological flow using flexible post array (49), Ting et al. devised a microfluidic arrays of rectangular blocks and flexible microposts which were subjected to arterial blood flow (Figure 1D) (40, 79). They measured the deflections of microposts caused by contracting platelet aggregates around the rectangular block, thereby quantifying the ensemble platelet contractile forces, in which myosin II, ADP activation and integrin GPIIb/IIIa were found to play key roles. Further, Myers et al. designed an array of fibrinogen-coated microdots patterned on a hydrogel substrate, where platelets adhering to the microdot extended filopodia to the adjacent microdot (68). The displacement of the microdots due to platelet contraction enabled quantification of platelet contractility in a cytometry manner. Evidently, the micropost array is advantageous to visualize and measure platelet contractility and can be extended to the diagnosis of reduced platelet contractility associated with bleeding conditions including Wiskott Aldrich Syndrome (WAS) (68) and Immune Thrombocytopenia (ITP) (82).

\section{Platelet-VWF Association}

In addition to the hemodynamic microenvironment, platelets interact with the circulatory components including the vessel walls, blood cells (red blood cells, T-cells) and plasma proteins (VWF, fibrinogen, tissue factor, collagen) (83). Particularly, VWF is central to primary hemostasis in mediating platelet recruitment to the damaged vascular subendothelium and subsequent platelet aggregation (84). The critical role of VWF in regulating platelet biomechanical adhesion and aggregation was investigated in numerous microfluidic systems. 
TABLE 1 | Summary of microfluidic models for platelet mechanobiology in thrombotic diseases.

\begin{tabular}{|c|c|c|c|c|c|c|}
\hline $\begin{array}{l}\text { Microfluidics } \\
\text { description }\end{array}$ & Fabrication method & Mechanical profile & Vasculature status & Perfusion sample & Functionality test & References \\
\hline
\end{tabular}

$\begin{aligned} & \text { Single layered simple structure microfluidics } \\ & \text { Straight channel }\end{aligned}$
Straight channel lithography

Pump-driven constant HUVECs w/wo TNF- $\alpha$ Recalcified human WB Thrombus formation or shear input and output stimulation platelet function assay

Pump-driven constant Primary HMVECs

Recalcified human WB Clot formation upon

shear input and output

diabetic complications

$(16,41,42)$

Pump-driven constant

flow input and

CGN/TF coated bare

Ladder network

Soft lithography

channel

geometry mediated

flow output

Bifurcation channel Soft lithography

Pump-driven constant

flow input and

geometry mediated

flow output

Junction with multiple Soft lithography

inlets and single outlet

Pump-driven constant HUVECs

flow input and output

2D stenosis channel Soft lithography

Pump-driven constant

flow input and

Non-coated bare

channel

geometry mediated

flow output

2D stenosis channel Aluminum vertical milling with non-uniform height molding; Soft lithography

Pressure-driven Type I CGN coated constant flow input and bare channel geometry mediated

flow output

Circular 3D stenosis channel

3D printing; Soft lithography

Pump-driven

flow input and

geometry mediated

flow output

Flexible micropost array Soft lithography

No external mechanical FNG or FNT coated

input array

HUVECS

(1)

Citrated human WB

Flow-mediated

blood-EC interaction

and thrombosis

Platelet-rich plasma

Platelet contractile

forces characterization

Gravity driven constant

flow input and

ECM molecules coated Human/mouse WB bare channel

Shear-dependent

platelet adhesion

geometry mediated

flow output

Pump-driven constant

flow input and

CGN or WWF coated

bare channel

Citrated human WB

Hemodynamic effect

on platelet contractility geometry mediated

flow output

\section{Three-layered microfluidics}

Assembled web network

Two chambers separated by porous

membrane layer

Top pressure actuation

chamber, middle

diaphragm, bottom

channel

Micropatterns printed

on microfluidic channel
Assembly soft lithography; Injection molding with Type 1 Collagen hydrogel

3D printing; Assembly soft lithography

Assembly soft lithography; injection molding

Assembly soft lithography micropattern with stamping; hydrogel casting
Gravity driven constant HUVECs only or

flow input and

geometry mediated flow pattern

cocultured with

HBVPCS/HUASMCs

Pump-driven constant flow input and output

Pneumatic peristaltic pump-driven flow

Pump-driven constant

flow and ECM mediated stiffness stimuli
HUVECs/HPMECs cocultured with alveolar epithelium

Type 1 CGN coated

FNG coated micropattern bare channel
Citrated human WB Angiogenesis and thrombosis; Flow-driven assembly of WWF fibers and its interaction with platelet

Recalcified human WB Platelet-endothelial dynamics in pulmonary thrombosis

Platelet thrombosis assay and antiplatelet drug screening

Citrated human WB/washed platelet

Platelet-rich plasma

Hemodynamic and ECM influences on platelet contractility

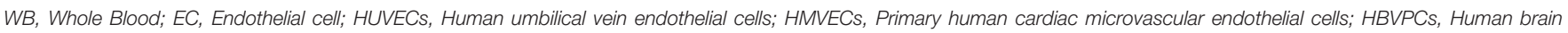

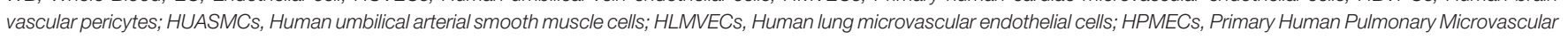
Endothelial Cells; ECM, Extracellular matrix; TNF- $\alpha$, tumor necrosis factor-alpha; WWF, von Willebrand Factor; TF, Tissue factor; FNG, Fibrinogen; CGN, Collagen. 
Notably, Dong et al. employed a commercial parallel plate flow chamber comprising a polycarbonate slab, silicon gasket and an endothelial cell-lined glass coverslip to study platelet GPIb $\alpha-V W F$ mediated platelet adhesion (85). Upon endothelial activation, platelets adhered to the released ultra-large VWF and formed extraordinary long 'beads-on-a-string'-like structure at a venous shear rate. Using the same parallel plate device, Padilla et al. proposed to reduce platelet-endothelial VWF interaction by exposing the VWF-A2 domain to ADAMTS13 under elongational shear (86). However, such parallel plate flow chamber cannot investigate more disturbed hemodynamic conditions due to its laminar flow profile.

Zheng et al. designed a complex endothelialized microvascular network (Figure 1E) (67). Such microfluidic model was fabricated by injection molding type I collagen between two plexiglass pieces and PDMS for stabilization. Zheng et al. then optimized the model to incorporate multiple vascular geometries including straight, grid, tortuous, stenosed channels (15) to investigate flow pattern-driven platelet-VWF interaction. The fluid dynamic profile near the bifurcation and vessel junctions enhanced VWF self-association, causing irreversible rapid platelet adhesion and aggregation. Westein et al. fabricated an atherosclerotic vessel mimicking microfluidic device with a halfcircular eccentric stenosis (20-80\% stenosis) and confirmed that plasma VWF strongly influenced the proaggregatory response (Figure 1F) (6). The VWF multimers were found to elongate with shear gradients and occupied a 15-times higher surface area in deceleration zones promoting extensive platelet aggregation. The significance of VWF was further supported by Kim et al. using a stenotic microfluidic model (87). As such, complex platforms with diverse geometries is beneficial to the study of thrombotic therapies targeting VWF self-association and the subsequent platelet responses (88).

\section{Platelet-Endothelium Interaction}

Endothelial expression of P-selectin, E-selectin, vascular cell adhesion molecule 1 (VCAM-1) and intercellular adhesion molecule 1 (ICAM-1) can regulate platelet activation and adhesion (83). Understanding the influence of the endothelium on platelet binding kinetics is essential to unravel the mechanism underlying platelet thrombus formation.

Ciciliano et al. employed a T-shape endothelialized microfluidic platform to recapitulate $\mathrm{FeCl}_{3}$-induced thrombosis in vitro (72). The platform is composed of a main channel where endothelial cells were cultured to form a confluent endothelium, and a side channel where $\mathrm{FeCl}_{3}$ was infused to join the main channel to create the blood- $\mathrm{FeCl}_{3}$ interface. The $\mathrm{FeCl}_{3}$ influx induced endothelial injury, followed by the binding between $\mathrm{Fe}^{3+}$ ions and negatively charged proteins, which lead to platelet activation and occlusive aggregation. Dupuy et al. developed a thromboinflammation model by using six parallel microchannels with fixed human umbilical vein endothelial cells (HUVECs) alignment (16). Tumor necrosis factor alpha (TNF- $\alpha$ ) treatment stimulated the expression of ICAM-1 and VCAM-1, and the secretion of VWF and tissue factor on the endothelium, leading to a dose-dependent increase in platelet-endothelial binding.
Further, Jain et al. utilized the same model and found that TNF- $\alpha$ activated HUVECs increased E-selectin expression (41).

Notably, Jain et al. exhibited a triple-layered human lung alveolus-on-chip to study the interplay between platelets, vessel wall, and blood flow (Figure 1G) (61). The model mainly contains an upper microchannel coated with primary human lung alveolar epithelial cells and a lower microchannel lined with HUVECs, separated by a thin and porous membrane. Remarkably, TNF- $\alpha$ and lipopolysaccharide endotoxin stimulated the alveolar epithelium and increased the pulmonary vascular permeability, resulting in enhanced ICAM-1 expression and platelet-endothelial binding.

\section{MICROFLUIDIC STANDARDIZATION}

Increased usages of shear-based microdevices by different laboratories for examining platelet functions lead to heterogenous device characteristics. Lack of standardization in microfluidic designs and operating methods complicates the result comparison and reproducibility, which impedes translation into both clinical practices and industrial settings $(89,90)$. The ISTH Biorheology subcommittee has advocated for standardization of flow chamber-based thrombus formation assays $(34,89,91,92)$. Requirements for microfluidic standardization can be extended to the following considerations.

(i) Structural design. To test the abnormalities in platelet mechanosensing, several microfluidic designs can be standardized: shear dependent platelet aggregation can be assessed in straight channels; shear gradient dependent thrombus development can be investigated in stenotic channels; and micropillar array can be standardized for platelet contractility measurements (5). Standardizations of these geometries and dimensions ensure unified microfluidics to be used for platelet function tests by different laboratories (93).

(ii) Surface treatment. Adhesive ligands should be considered carefully to induce platelet adhesion, aggregation and contraction (94-97). Specifically, concentration, duration of treatment, molecular composition, coating procedure of the adhesive proteins needs to be specified for standard usage (95). Moreover, the source, type, passage number, culture medium, seeding procedure, culture duration, inflammatory stimulation of cell-derived surface should be considered thoroughly.

(iii) Sample preparation and perfusion. It is important to ensure appropriate handling of the perfusion samples which include whole blood and washed platelets. Source of blood samples, procedure and handling of blood collection, storage, and the use of anticoagulants are to be standardized (94).

(iv) Hemodynamic settings. Microfluidic device materials, tubing dimensions, perfusion pumps and choice of bulk shear rates are essential when conducting flow tests (98). Standardizations of these hemodynamic settings including the inertial forces and Reynolds number within the 
TABLE 2 | Commercialized microfluidic slides for laboratory research.

\begin{tabular}{|c|c|c|c|c|c|c|c|}
\hline Flow chamber & Surface treatment & Vasculature status & $\begin{array}{l}\text { Shear rate } \\
\left(\text { dyne } / \mathrm{cm}^{2}\right)\end{array}$ & $\begin{array}{l}\text { Perfusion } \\
\text { sample }\end{array}$ & Investigation & References & $\begin{array}{l}\text { Representative } \\
\text { image }\end{array}$ \\
\hline Ibidi $\mu$-slide V| $\mathrm{I}^{0.4}$ & Collagen /thrombin & No ECs & Static & Washed platelet & $\begin{array}{l}\text { Platelet activation } \\
\text { plasminogen activator } \\
\text { inhibitor } 1\end{array}$ & (99) & Ibidi $\mu$-Slide $\mathrm{VI}^{0.4}$ \\
\hline Ibidi $\mu$-slide V| $\mathrm{I}^{0.4}$ & No specific coating & HPAECS & $150 s^{-1}$ & Mouse/human WB & $\begin{array}{l}\text { Platelet activation lethal } \\
\text { sepsis }\end{array}$ & $(100)$ & \\
\hline Ibidi $\mu$-slide $\mathrm{VI}^{0.4}$ & $\begin{array}{l}\text { Human Fc-podoplanin } \\
\text { fusion protein }\end{array}$ & LECs /HUVECs & $50-1,350 \mathrm{~s}^{-1}$ & Mouse/human WB & $\begin{array}{l}\text { Effect of } \\
\text { CLEC-2-podoplanin } \\
\text { interactions on Platelet } \\
\text { adhesion }\end{array}$ & (101) & Ibidi $\mu$-Slide $\mathrm{VI}^{0.1}$ \\
\hline Ibidi $\mu$-slides & Fibrinogen & No ECs & Static & Human WB & $\begin{array}{l}\text { Platelet adhesion and } \\
\text { thrombo-inflammation }\end{array}$ & (102) & \\
\hline $\begin{array}{l}\text { Ibidi } \mu \text {-slides/Biotechs } \\
\text { FCS2 flow chamber }\end{array}$ & Collagen type 1 & No ECs & $200-1,000 \mathrm{~s}^{-1}$ & Washed platelets & $\begin{array}{l}\text { Platelet-monocyte } \\
\text { interactions }\end{array}$ & (103) & Cellix Vena8 Fluoro+ \\
\hline $\begin{array}{l}\text { Cellix Vena8 Fluoro+ } \\
\text { biochip }\end{array}$ & Fibrillar collagen & No ECs & $200-1,000 \mathrm{~s}^{-1}$ & $\begin{array}{l}\text { Washed } \\
\text { platelet/Human } \\
\text { WB }\end{array}$ & $\begin{array}{l}\text { Effects of } \beta \text { amyloid } \\
\text { peptides on platelet }\end{array}$ & (104) & \\
\hline $\begin{array}{l}\text { Cellix Vena8 Fluoro + } \\
\text { biochip }\end{array}$ & Collagen type 1 & No ECs & $90 s^{-1}$ & $\begin{array}{l}\text { Platelet-rich } \\
\text { plasma }\end{array}$ & $\begin{array}{l}\text { Effect of omega- } 3 \text { fatty } \\
\text { acids on platelet } \\
\text { aggregation }\end{array}$ & (105) & \\
\hline Cellix Vena8 biochips & Collagen/fibrinogen & No ECs & 0.5 dyne $/ \mathrm{cm}^{2}$ & $\begin{array}{l}\text { Platelet-rich } \\
\text { plasma/human } \\
\text { WB }\end{array}$ & $\begin{array}{l}\text { Effect of prostaglandin on } \\
\text { platelet aggregation }\end{array}$ & (106) & $\begin{array}{l}\text { ena8 } \\
\text { elial+ biochips }\end{array}$ \\
\hline $\begin{array}{l}\text { Cellix Vena } 8 \text { Endothelial + } \\
\text { biochips }\end{array}$ & Fibronectin & HCAECs & 0.5 dyne $/ \mathrm{cm}^{2}$ & Washed platelets & $\begin{array}{l}\text { Effect of plasma protein on } \\
\text { platelet-endothelium } \\
\text { crosstalk }\end{array}$ & $(107)$ & \\
\hline $\begin{array}{l}\text { Cellix Vena8 Fluoro+ } \\
\text { biochip }\end{array}$ & Collagen type 1 & No ECs & $67.5 \mathrm{dyne} / \mathrm{cm}^{2}$ & Human WB & $\begin{array}{l}\text { Effect of Gut Microbial } \\
\text { Metabolite TMAO on } \\
\text { platelet and thrombosis }\end{array}$ & (99) & \\
\hline Cellix Vena8 biochips & Fibrinogen/collagen & $\begin{array}{l}\text { HUVECs w/wo TNF- } \alpha \\
\text { stimulation }\end{array}$ & 0.3 dyne $/ \mathrm{cm}^{2}$ & $\begin{array}{l}\text { Unwashed } \\
\text { platelet-rich } \\
\text { plasma/washed } \\
\text { platelet }\end{array}$ & $\begin{array}{l}\text { Platelet activation and } \\
\text { adhesion to diseased } \\
\text { endothelium }\end{array}$ & $(100)$ & $\begin{array}{l}\text { Glycotech Flow } \\
\text { Chamber }\end{array}$ \\
\hline $\begin{array}{l}\text { Cellix Vena8 Fluoro+ } \\
\text { biochip }\end{array}$ & Equine tendon collagen & No ECs & 10 dyne/cm² & $\begin{array}{l}\text { Platelet-rich } \\
\text { plasma }\end{array}$ & $\begin{array}{l}\text { Effect of legacy } \\
\text { perfluoroalkyl substances } \\
\text { on platelet dynamics }\end{array}$ & (101) & \\
\hline
\end{tabular}




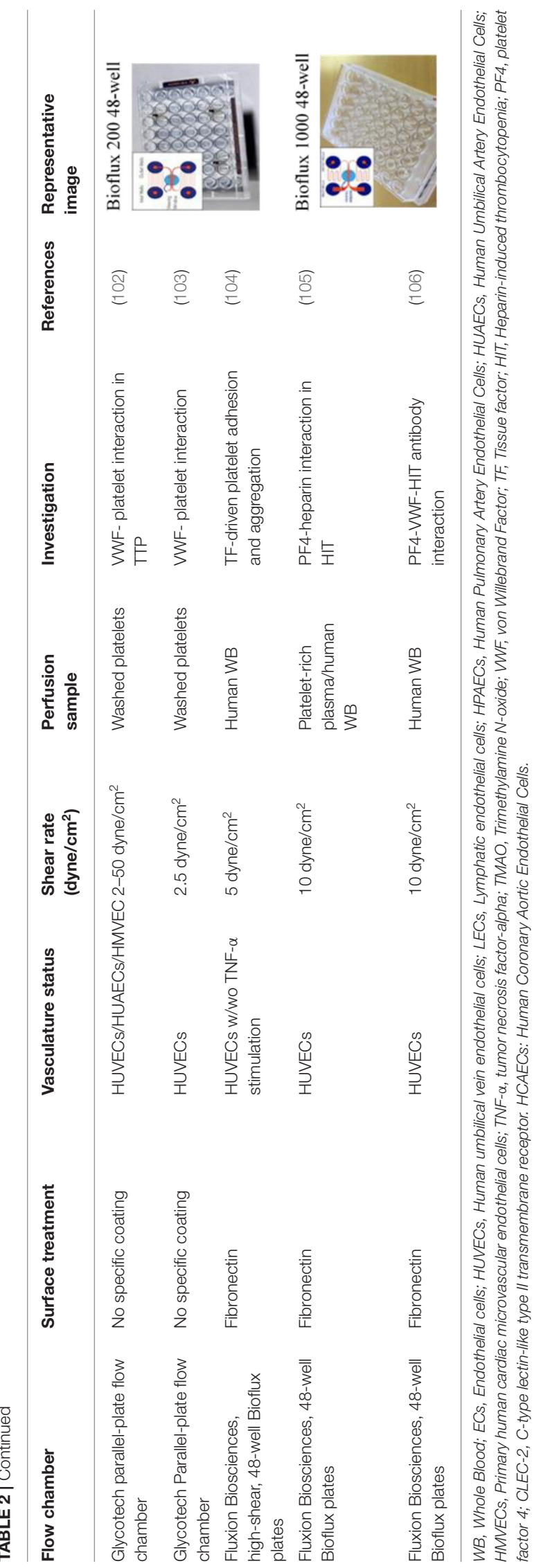

microfluidic systems will allow more certain conclusions to be extrapolated (93).

(v) Visualization and quantification methods. Standardization of microscopy-based analytical approaches can be based on different stages of thrombus formation indicated by platelet adhesion receptors, activated ligand expression and aggregate volume. The types, sources, and concentrations of fluorescent stains and antibodies should be specified to control adverse chemical and phototoxic effect on platelet functions. The method of quantification of the fluorescent microscopy data needs to be standardized to allow more transparency in obtained results.

\section{COMMERCIALLY AVAILABLE MICROFLUIDICS FOR LABORATORY USE}

The geometry of microfluidic devices is of the utmost relevance to their application in platelet function studies and their extension to antiplatelet drug screening platforms. Commercially available microfluidic systems have been able to standardize more simple, quasi-2D planar geometries but are still limited by their inability to create complex geometry in a high-throughput manner. A summary of studies looking at platelet biology in thrombosis using commercialized microfluidic slides is presented in Table 2.

For effective commercialization of more complicated microfluidic structures, the fabrication protocol need to be high-throughput, robust, reproducible and cost-efficient (108). Such fabrication techniques including nanoimprint lithography, 3D printing and anodic aluminum oxidation are promising in supporting rapid-prototyping of highly tailorable complex microfluidics with replicable specifications $(90,108)$. With increased standardization and advancements in the commercial capacity, biomimetic microfluidic systems will accelerate novel antiplatelet therapeutic development and have great potential in patient screening (109).

\section{CONCLUSION}

Emerging microfluidic technologies have advanced our understanding of platelet mechanobiology and its role in hemostasis and thrombosis. The ease of manufacturing complex geometries, versatility and the high throughput of microfluidic experiments have enabled researchers to investigate how platelets respond to their hemodynamic microenvironment and interact with the circulatory system in a well-controlled biomechanical milieu. The varieties in designs, fabrications, operation procedures and analytical approaches of customized microfluidics have urged the standardization need for reproducibility. The increasing use of commercially available microfluidics accelerates the translation of platelet mechanobiology to pre-clinical and industrial applications. In the next decade, we foresee microfluidic technologies to be used for patient-specific disease management, diagnosis, as well as antiplatelet drug screening. 


\section{AUTHOR CONTRIBUTIONS}

$\mathrm{YZ}$ and LJ conceived the study and wrote the manuscript. VC, $\mathrm{RP}$, and SR co-wrote the manuscript. HL and LJ provided critical comments, suggestions, and text. LJ designed and supervised the study. All authors contributed to the article and approved the submitted version.

\section{FUNDING}

This work was supported by the Australian Research Council (ARC) Discovery Project (DP200101970 - LJ), the National Health and Medical Research Council (NHMRC) of Australia Ideas Grant (APP2003904 - LJ), NSW Cardiovascular

\section{REFERENCES}

1. Bagot CN, Arya R. Virchow and his triad: a question of attribution. $\mathrm{Br} \mathrm{J}$ Haematol. (2008) 143:180-90. doi: 10.1111/j.1365-2141.2008.07323.x

2. Chen Y, Ju LA. Biomechanical thrombosis: the dark side of force and dawn of mechano-medicine. Stroke Vasc Neurol. (2020) 5:185-97. doi: 10.1136/svn-2019-000302

3. Lakshmanan HHS, Shatzel JJ, Olson SR, McCarty OJT, Maddala J. Modeling the effect of blood vessel bifurcation ratio on occlusive thrombus formation. Comput Methods Biomech Biomed Eng. (2019) 22:972-80. doi: 10.1080/10255842.2019.1610744

4. Nesbitt WS, Westein E, Tovar-Lopez FJ, Tolouei E, Mitchell A, Fu J, et al. A shear gradient-dependent platelet aggregation mechanism drives thrombus formation. Nat Med. (2009) 15:665-73. doi: 10.1038/nm.1955

5. Zhang C, Neelamegham S. Application of microfluidic devices in studies of thrombosis and hemostasis. Platelets. (2017) 28:434-40. doi: 10.1080/09537104.2017.1319047

6. Westein E, van der Meer AD, Kuijpers MJ, Frimat JP, van den Berg A, Heemskerk JW. Atherosclerotic geometries exacerbate pathological thrombus formation poststenosis in a von Willebrand factor-dependent manner. Proc Natl Acad Sci USA. (2013) 110:1357-62. doi: 10.1073/pnas.1209905110

7. Varble N, Trylesinski G, Xiang J, Snyder K, Meng H. Identification of vortex structures in a cohort of 204 intracranial aneurysms. J R Soc Interface. (2017) 14:20170021. doi: 10.1098/rsif.2017.0021

8. Ha H, Ziegler M, Welander M, Bjarnegård N, Carlhäll C-J, Lindenberger $\mathrm{M}$, et al. Age-related vascular changes affect turbulence in aortic blood flow. Front Physiol. (2018) 9:36. doi: 10.3389/fphys.2018.00036

9. Ruggeri ZM. Platelets in atherothrombosis. Nat Med. (2002) 8:1227-34. doi: $10.1038 / \mathrm{nm} 1102-1227$

10. Jackson SP. Arterial thrombosis-insidious, unpredictable and deadly. Nat Med. (2011) 17:1423-36. doi: 10.1038/nm.2515

11. Osdoit S, Rosa JP. Fibrin clot retraction by human platelets correlates with alpha(IIb)beta(3) integrin-dependent protein tyrosine dephosphorylation. $J$ Biol Chem. (2001) 276:6703-10. doi: 10.1074/jbc.M008945200

12. Hansen CE, Qiu Y, McCarty OJT, Lam WA. Platelet mechanotransduction. Annu Rev Biomed Eng. (2018) 20:253-75. doi: 10.1146/annurev-bioeng-062117-121215

13. McFadyen JD, Schaff M, Peter K. Current and future antiplatelet therapies: emphasis on preserving haemostasis. Nat Rev Cardiol. (2018) 15:181-91. doi: $10.1038 /$ nrcardio. 2017.206

14. Lin J, Sorrells MG, Lam WA, Neeves KB. Physical forces regulating hemostasis and thrombosis: vessels, cells, and molecules in illustrated review. Res Pract Thromb Haemost. (2021) 5:e12548. doi: 10.1002/rth2.12548

15. Zheng Y, Chen J, Lopez JA. Flow-driven assembly of VWF fibres and webs in in vitro microvessels. Nat Commun. (2015) 6:7858. doi: $10.1038 /$ ncomms8858
Capacity Building Program (Early-Mid Career Researcher Grant - LJ), Sydney Research Accelerator prize (SOAR - LJ), and Ramaciotti Foundations Health Investment Grant (2020HIG76 - LJ). LJ is an ARC DECRA fellow (DE190100609) and National Heart Foundation Future Leader fellow (FLF2 105863).

\section{ACKNOWLEDGMENTS}

We thank Yunduo Zhao, Fangyuan Zhou, and Fengtao Jiang for the helpful discussion. We acknowledge Nadia Court and Jacky He at the Research Prototype Foundry, Simon Ringer and Gwenaelle Proust at the Sydney Manufacturing Hub for the support of lab startup.

16. Dupuy A, Hagimola L, Mgaieth NSA, Houlahan CB, Preketes-Tardiani RE, Coleman PR, et al. Thromboinflammation model-on-a-chip by whole blood microfluidics on fixed human endothelium. Diagnostics. (2021) 11:203. doi: 10.3390/diagnostics11020203

17. Lam WA, Chaudhuri O, Crow A, Webster KD, Li TD, Kita A, et al. Mechanics and contraction dynamics of single platelets and implications for clot stiffening. Nat Mater. (2011) 10:61-6. doi: 10.1038/nmat2903

18. Zhang W, Deng W, Zhou L, Xu Y, Yang W, Liang X, et al. Identification of a juxtamembrane mechanosensitive domain in the platelet mechanosensor glycoprotein Ib-IX complex. Blood. (2015) 125:562-9. doi: 10.1182/blood-2014-07-589507

19. Chen Y, Ju LA, Zhou F, Liao J, Xue L, Su QP, et al. An integrin alphaIIbbeta3 intermediate affinity state mediates biomechanical platelet aggregation. Nat Mater. (2019) 18:760-69. doi: 10.1038/s41563-019-0323-6

20. Zhu C, Chen Y, Ju LA. Dynamic bonds and their roles in mechanosensing. Curr Opin Chem Biol. (2019) 53:88-97. doi: 10.1016/j.cbpa.2019.08.005

21. Colace TV, Tormoen GW, McCarty OJ, Diamond SL. Microfluidics and coagulation biology. Annu Rev Biomed Eng. (2013) 15:283-303. doi: 10.1146/annurev-bioeng-071812-152406

22. Furie B, Furie BC. Thrombus formation in vivo. J Clin Invest. (2005) 115:3355-62. doi: 10.1172/JCI26987

23. Festing S, Wilkinson R. The ethics of animal research. Talking Point on the use of animals in scientific research. EMBO Rep. (2007) 8:526-30. doi: 10.1038/sj.embor.7400993

24. Bracken MB. Why animal studies are often poor predictors of human reactions to exposure. $J$ R Soc Med. (2009) 102:120-2. doi: 10.1258 /jrsm.2008.08k033

25. Van Kruchten R, Cosemans JM, Heemskerk JW. Measurement of whole blood thrombus formation using parallel-plate flow chambers - a practical guide. Platelets. (2012) 23:229-42. doi: 10.3109/09537104.2011.630848

26. Giorgio TD, Hellums JD. A cone and plate viscometer for the continuous measurement of blood platelet activation. Biorheology. (1988) 25:605-24. doi: 10.3233/BIR-1988-25402

27. Solomon C, Ranucci M, Hochleitner G, Schöchl H, Schlimp CJ. Assessing the methodology for calculating platelet contribution to clot strength (platelet component) in thromboelastometry and thrombelastography. Anesth Analg. (2015) 121:868-78. doi: 10.1213/ANE.000000000000 0859

28. Branchford BR, Ng CJ, Neeves KB, Di Paola J. Microfluidic technology as an emerging clinical tool to evaluate thrombosis and hemostasis. Thromb Res. (2015) 136:13-9. doi: 10.1016/j.thromres.2015.05.012

29. Herbig BA, Yu X, Diamond SL. Using microfluidic devices to study thrombosis in pathological blood flows. Biomicrofluidics. (2018) 12:042201. doi: 10.1063/1.5021769

30. Westein E, de Witt S, Lamers M, Cosemans JM, Heemskerk JW. Monitoring in vitro thrombus formation with novel microfluidic devices. Platelets. (2012) 23:501-9. doi: 10.3109/09537104.2012.709653 
31. Brummel-Ziedins KE, Wolberg AS. Global assays of hemostasis. Curr Opin Hematol. (2014) 21:395. doi: 10.1097/MOH.0000000000000074

32. Lipets EN, Ataullakhanov FI. Global assays of hemostasis in the diagnostics of hypercoagulation and evaluation of thrombosis risk. Thromb J. (2015) 13:1-15. doi: 10.1186/s12959-015-0038-0

33. Rubenstein DA, Yin W, Frame MD. Biofluid Mechanics : An Introduction to Fluid Mechanics, Macrocirculation, and Microcirculation, 2nd ed. Amsterdam; Boston, MA: Elsevier;Academic Press (2015). p. xii, 531. ISBN: 9780128009444

34. Zwaginga JJ, Nash G, King MR, Heemskerk JW, Frojmovic M, Hoylaerts MF, et al. Flow-based assays for global assessment of hemostasis. Part 1: Biorheologic considerations. J Thromb Haemost. (2006) 4:2486-7. doi: 10.1111/j.1538-7836.2006.02177.x

35. Jackson SP, Darbousset R, Schoenwaelder SM. Thromboinflammation: challenges of therapeutically targeting coagulation and other host defense mechanisms. Blood. (2019) 133:906-18. doi: 10.1182/blood-2018-11-882993

36. Gando S. Microvascular thrombosis and multiple organ dysfunction syndrome. Crit Care Med. (2010) 38:S35-42. doi: 10.1097/CCM.0b013e3181c9e31d

37. McFadyen JD, Stevens H, Peter K. The emerging threat of (micro)thrombosis in covid-19 and its therapeutic implications. Circ Res. (2020) 127:571-87. doi: 10.1161/CIRCRESAHA.120.317447

38. Bray MA, Sartain SE, Gollamudi J, Rumbaut RE. Microvascular thrombosis: experimental and clinical implications. Transl Res. (2020) 225:105-30. doi: 10.1016/j.trsl.2020.05.006

39. Tsai M, Kita A, Leach J, Rounsevell R, Huang JN, Moake J, et al. In vitro modeling of the microvascular occlusion and thrombosis that occur in hematologic diseases using microfluidic technology. J Clin Investig. (2012) 122:408-18. doi: 10.1172/JCI58753

40. Ting LH, Feghhi S, Taparia N, Smith AO, Karchin A, Lim E, et al. Contractile forces in platelet aggregates under microfluidic shear gradients reflect platelet inhibition and bleeding risk. Nat Commun. (2019) 10:1204. doi: 10.1038/s41467-019-09150-9

41. Jain A, van der Meer AD, Papa AL, Barrile R, Lai A, Schlechter BL, et al. Assessment of whole blood thrombosis in a microfluidic device lined by fixed human endothelium. Biomed Microdev. (2016) 18:73. doi: 10.1007/s10544-016-0095-6

42. Albers HJ, Passier R, van den Berg A, van der Meer AD. Automated analysis of platelet aggregation on cultured endothelium in a microfluidic chip perfused with human whole blood. Micromachines. (2019) 10:781. doi: $10.3390 / \mathrm{mi} 10110781$

43. Ju L, Chen Y, Zhou F, Lu H, Cruz MA, Zhu C. Von Willebrand factor-A1 domain binds platelet glycoprotein Ibalpha in multiple states with distinctive force-dependent dissociation kinetics. Thromb Res. (2015) 136:606-12. doi: 10.1016/j.thromres.2015.06.019

44. de Witt SM, Swieringa F, Cavill R, Lamers MM, van Kruchten R, Mastenbroek T, et al. Identification of platelet function defects by multiparameter assessment of thrombus formation. Nat Commun. (2014) 5:4257. doi: $10.1038 /$ ncomms5257

45. Jenny L, Melmer A, Laimer M, Hardy ET, Lam WA, Schroeder V. Diabetes affects endothelial cell function and alters fibrin clot formation in a microvascular flow model: a pilot study. Diab Vasc Dis Res. (2020) 17:1479164120903044. doi: 10.1177/1479164120903044

46. Tovar-Lopez FJ, Rosengarten G, Westein E, Khoshmanesh K, Jackson SP, Mitchell A, et al. A microfluidics device to monitor platelet aggregation dynamics in response to strain rate micro-gradients in flowing blood. Lab Chip. (2010) 10:291-302. doi: 10.1039/B916757A

47. Zilberman-Rudenko J, Sylman JL, Lakshmanan HHS, McCarty OJT, Maddala J. Dynamics of blood flow and thrombus formation in a multi-bypass microfluidic ladder network. Cell Mol Bioeng. (2017) 10:16-29. doi: 10.1007/s12195-016-0470-7

48. Gray KM, Stroka KM. Vascular endothelial cell mechanosensing: new insights gained from biomimetic microfluidic models. Semin Cell Dev Biol. (2017) 71:106-17. doi: 10.1016/j.semcdb.2017.06.002

49. Liang XM, Han SJ, Reems JA, Gao D, Sniadecki NJ. Platelet retraction force measurements using flexible post force sensors. Lab Chip. (2010) 10:991-8. doi: $10.1039 / \mathrm{b} 918719 \mathrm{~g}$
50. Yin W, Shanmugavelayudam SK, Rubenstein DA. The effect of physiologically relevant dynamic shear stress on platelet and endothelial cell activation. Thromb Res. (2011) 2011/03/01/;127:235-41. doi: 10.1016/j.thromres.2010.11.021

51. Sheriff J, Soares JS, Xenos M, Jesty J, Bluestein D. Evaluation of shearinduced platelet activation models under constant and dynamic shear stress loading conditions relevant to devices. Ann Biomed Eng. (2013) 41:1279-96. doi: 10.1007/s10439-013-0758-x

52. Zhao YC, Vatankhah P, Goh T, Michelis R, Kyanian K, Zhang Y, et al. Hemodynamic analysis for stenosis microfluidic model of thrombosis with refined computational fluid dynamics simulation. Sci Rep. (2021) 11:6875. doi: 10.1038/s41598-021-86310-2

53. Casa LDC, Deaton DH, Ku DN. Role of high shear rate in thrombosis. J Vasc Surg. (2015) 61:1068-80. doi: 10.1016/j.jvs.2014.12.050

54. Bark DL Jr., Para AN, Ku DN. Correlation of thrombosis growth rate to pathological wall shear rate during platelet accumulation. Biotechnol Bioeng. (2012) 109:2642-50. doi: 10.1002/bit.24537

55. Szydzik C, Brazilek RJ, Akbaridoust F, de Silva C, Moon M, Marusic I, et al. Active micropump-mixer for rapid antiplatelet drug screening in whole blood. Anal Chem. (2019) 91:10830-9. doi: 10.1021/acs.analchem. $9 \mathrm{~b} 02486$

56. Costa PF, Albers HJ, Linssen JEA, Middelkamp HHT, van der Hout L, Passier $\mathrm{R}$, et al. Mimicking arterial thrombosis in a 3D-printed microfluidic in vitro vascular model based on computed tomography angiography data. Lab Chip. (2017) 17:2785-92. doi: 10.1039/C7LC00202E

57. Menon NV, Su C, Pang KT, Phua ZJ, Tay HM, Dalan R, et al. Recapitulating atherogenic flow disturbances and vascular inflammation in a perfusable 3D stenosis model. Biofabrication. (2020) 12:045009. doi: 10.1088/1758-5090/aba501

58. Lee EJ, Cines DB, Gernsheimer T, Kessler C, Michel M, Tarantino MD, et al. Thrombocytopenia following Pfizer and Moderna SARS-CoV-2 vaccination. Am J Hematol. (2021) 96:534-7. doi: 10.1002/ajh.26132

59. Mannino RG, Myers DR, Ahn B, Wang Y, Margo R, Gole H, et al. Doit-yourself in vitro vasculature that recapitulates in vivo geometries for investigating endothelial-blood cell interactions. Sci Rep. (2015) 5:12401. doi: $10.1038 /$ srep 12401

60. Hesh CA, Qiu Y, Lam WA. Vascularized microfluidics and the blood-endothelium interface. Micromachines. (2019) 11:18. doi: $10.3390 / \mathrm{mi} 11010018$

61. Jain A, Barrile R, van der Meer AD, Mammoto A, Mammoto T, De Ceunynck $\mathrm{K}$, et al. Primary human lung alveolus-on-a-chip model of intravascular thrombosis for assessment of therapeutics. Clin Pharmacol Ther. (2018) 103:332-40. doi: 10.1002/cpt.742

62. Qiu Y, Ahn B, Sakurai Y, Hansen CE, Tran R, Mimche PN, et al. Microvasculature-on-a-chip for the long-term study of endothelial barrier dysfunction and microvascular obstruction in disease. Nat Biomed Eng. (2018) 2:453-63. doi: 10.1038/s41551-018-0224-z

63. Liu X, Fang J, Huang S, Wu X, Xie X, Wang J, et al. Tumor-on-a-chip: from bioinspired design to biomedical application. Microsyst Nanoeng. (2021) 7:50. doi: 10.1038/s41378-021-00277-8

64. Sosa-Hernandez JE, Villalba-Rodriguez AM, Romero-Castillo KD, AguilarAguila-Isaias MA, Garcia-Reyes IE, Hernandez-Antonio A, et al. Organs-ona-chip module: a review from the development and applications perspective. Micromachines. (2018) 9:536. doi: 10.3390/mi9100536

65. Zhang YS, Davoudi F, Walch P, Manbachi A, Luo X, Dell'Erba V, et al. Bioprinted thrombosis-on-a-chip. Lab Chip. (2016) 16:4097-105. doi: 10.1039/C6LC00380J

66. Gutierrez E, Petrich BG, Shattil SJ, Ginsberg MH, Groisman A, Kasirer-Friede A. Microfluidic devices for studies of shear-dependent platelet adhesion. Lab Chip. (2008) 8:1486-95. doi: 10.1039/b804 $795 \mathrm{~b}$

67. Zheng Y, Chen J, Craven M, Choi NW, Totorica S, Diaz-Santana A, et al. In vitro microvessels for the study of angiogenesis and thrombosis. Proc Natl Acad Sci USA. (2012) 109:9342-7. doi: 10.1073/pnas.1201240109

68. Myers DR, Qiu Y, Fay ME, Tennenbaum M, Chester D, Cuadrado J, et al. Single-platelet nanomechanics measured by high-throughput cytometry. Nat Mater. (2017) 16:230-35. doi: 10.1038/nmat4772 
69. Sakurai Y, Hardy ET, Ahn B, Tran R, Fay ME, Ciciliano JC, et al. A microengineered vascularized bleeding model that integrates the principal components of hemostasis. Nat Commun. (2018) 9:509. doi: 10.1038/s41467-018-02990-x

70. Conant CG, Schwartz MA, Beecher JE, Rudoff RC, Ionescu-Zanetti C, Nevill JT. Well plate microfluidic system for investigation of dynamic platelet behavior under variable shear loads. Biotechnol Bioeng. (2011) 108:2978-87. doi: 10.1002/bit.23243

71. Jain A, Graveline A, Waterhouse A, Vernet A, Flaumenhaft R, Ingber DE. A shear gradient-activated microfluidic device for automated monitoring of whole blood haemostasis and platelet function. Nat Commun. (2016) 7:10176. doi: 10.1038/ncomms10176

72. Ciciliano JC, Sakurai Y, Myers DR, Fay ME, Hechler B, Meeks S, et al. Resolving the multifaceted mechanisms of the ferric chloride thrombosis model using an interdisciplinary microfluidic approach. Blood. (2015) 126:817-24. doi: 10.1182/blood-2015-02-628594

73. Li M, Ku DN, Forest CR. Microfluidic system for simultaneous optical measurement of platelet aggregation at multiple shear rates in whole blood. Lab Chip. (2012) 12:1355-62. doi: 10.1039/c2lc21145a

74. Chiu JJ, Chien S. Effects of disturbed flow on vascular endothelium: pathophysiological basis and clinical perspectives. Physiol Rev. (2011) 91:327-87. doi: 10.1152/physrev.00047.2009

75. Rana A, Westein E, Niego B, Hagemeyer CE. Shear-dependent platelet aggregation: mechanisms and therapeutic opportunities. Front Cardiovasc Med. (2019) 6:141. doi: 10.3389/fcvm.2019.00141

76. Li XJ, Li PC. Contraction study of a single cardiac muscle cell in a microfluidic chip. Methods Mol Biol. (2006) 321:199-225. doi: 10.1385/1-59259-997-4:199

77. Muthard RW, Diamond SL. Blood clots are rapidly assembled hemodynamic sensors: flow arrest triggers intraluminal thrombus contraction. Arterioscler Thromb Vasc Biol. (2012) 32:2938-45. doi: 10.1161/ATVBAHA.112.300312

78. Muthard RW, Diamond SL. Side view thrombosis microfluidic device with controllable wall shear rate and transthrombus pressure gradient. Lab Chip. (2013) 13:1883-91. doi: 10.1039/c3lc41332b

79. Ting L, Feghhi S, Karchin A, Tooley W, White NJ, Sniadecki N. Clot-on-a-chip: a microfluidic device to study platelet aggregation and contractility under shear. Blood. (2013) 122:2363-63. doi: 10.1182/blood.V122.21.2363.2363

80. Chen Z, Lu J, Zhang C, Hsia I, Yu X, Marecki L, et al. Microclot array elastometry for integrated measurement of thrombus formation and clot biomechanics under fluid shear. Nat Commun. (2019) 10:2051. doi: 10.1038/s41467-019-10067-6

81. van Rooij BJM, Zavodszky G, Hoekstra AG, Ku DN. Biorheology of occlusive thrombi formation under high shear: in vitro growth and shrinkage. Sci Rep. (2020) 10:18604. doi: 10.1038/s41598-020-74518-7

82. Oshinowo OT, Copeland R, Bennett CM, Lam WA, Myers DR. Platelet contraction force as a biophysical biomarker for bleeding risk in patients with immune thrombocytopenia. Blood. (2018) 132(Suppl. 1):517. doi: 10.1182/blood-2018-99-115292

83. Koupenova M, Clancy L, Corkrey HA, Freedman JE. Circulating platelets as mediators of immunity, inflammation, and thrombosis. Circ Res. (2018) 122:337-51. doi: 10.1161/CIRCRESAHA.117.310795

84. Peyvandi F, Garagiola I, Baronciani L. Role of von Willebrand factor in the haemostasis. Blood Transfus. (2011) 9(Suppl. 2):s3-8. doi: 10.2450/2011.002S

85. Dong JF, Moake JL, Nolasco L, Bernardo A, Arceneaux W, Shrimpton CN, et al. ADAMTS-13 rapidly cleaves newly secreted ultralarge von Willebrand factor multimers on the endothelial surface under flowing conditions. Blood. (2002) 100:4033-9. doi: 10.1182/blood-2002-05-1401

86. Padilla A, Moake JL, Bernardo A, Ball C, Wang Y, Arya M, et al. P-selectin anchors newly released ultralarge von Willebrand factor multimers to the endothelial cell surface. Blood. (2004) 103:2150-6. doi: 10.1182/blood-2003-08-2956

87. Kim DA, Ashworth KJ, Di Paola J, Ku DN. Platelet alpha-granules are required for occlusive high-shear-rate thrombosis. Blood Adv. (2020) 4:3258267. doi: 10.1182/bloodadvances.2020002117

88. Chung DW, Chen J, Ling M, Fu X, Blevins T, Parsons S, et al. High-density lipoprotein modulates thrombosis by preventing von Willebrand factor self-association and subsequent platelet adhesion. Blood. (2016) 127:637-45. doi: 10.1182/blood-2014-09-5 99530

89. Roest M, Reininger A, Zwaginga JJ, King MR, Heemskerk JW, Biorheology Subcommittee of the SSCotI. Flow chamber-based assays to measure thrombus formation in vitro: requirements for standardization. J Thromb Haemost. (2011) 9:2322-4. doi: 10.1111/j.1538-7836.2011. 04492.x

90. Chiu DT, deMello AJ, Di Carlo D, Doyle PS, Hansen C, Maceiczyk RM, et al. Small but perfectly formed? Successes, challenges, and opportunities for microfluidics in the chemical and biological sciences. Chem. (2017) 2:201-23. doi: 10.1016/j.chempr.2017.01.009

91. Neeves KB, McCarty OJ, Reininger AJ, Sugimoto M, King MR, Biorheology Subcommittee of the SSCotI. Flow-dependent thrombin and fibrin generation in vitro: opportunities for standardization: communication from SSC of the ISTH. J Thromb Haemost. (2014) 12:418-20. doi: 10.1111/jth. 12482

92. Zwaginga JJ, Sakariassen KS, Nash G, King MR, Heemskerk JW, Frojmovic $\mathrm{M}$, et al. Flow-based assays for global assessment of hemostasis. Part 2: current methods and considerations for the future. J Thromb Haemost. (2006) 4:2716-7. doi: 10.1111/j.1538-7836.2006.02178.x

93. McCarty OJ, Ku D, Sugimoto M, King MR, Cosemans JM, Neeves KB, et al. Dimensional analysis and scaling relevant to flow models of thrombus formation: communication from the SSC of the ISTH. J Thromb Haemost. (2016) 14:619-22. doi: 10.1111/jth.13241

94. Mangin PH, Gardiner EE, Nesbitt WS, Kerrigan SW, Korin N, Lam WA, et al. In vitro flow based systems to study platelet function and thrombus formation: recommendations for standardization: communication from the SSC on Biorheology of the ISTH. J Thromb Haemost. (2020) 18:748-52. doi: $10.1111 /$ jth. 14717

95. Heemskerk JW, Sakariassen KS, Zwaginga JJ, Brass LF, Jackson SP, Farndale RW, et al. Collagen surfaces to measure thrombus formation under flow: possibilities for standardization. J Thromb Haemost. (2011) 9:856-8. doi: 10.1111/j.1538-7836.2011.04230.x

96. Orvim U, Roald HE, Stephens RW, Roos N, Sakariassen KS. Tissue factorinduced coagulation triggers platelet thrombus formation as efficiently as fibrillar collagen at arterial blood flow conditions. Arterioscler Thromb. (1994) 14:1976-83. doi: 10.1161/01.ATV.14.12.1976

97. Okorie UM, Denney WS, Chatterjee MS, Neeves KB, Diamond SL. Determination of surface tissue factor thresholds that trigger coagulation at venous and arterial shear rates: amplification of $100 \mathrm{fM}$ circulating tissue factor requires flow. Blood. (2008) 111:3507-13. doi: 10.1182/blood-2007-08-1 06229

98. Coenen DM, Mastenbroek TG, Cosemans J. Platelet interaction with activated endothelium: mechanistic insights from microfluidics. Blood. (2017) 130:2819-28. doi: 10.1182/blood-2017-04-780825

99. Morrow GB, Whyte CS, Mutch NJ. Functional plasminogen activator inhibitor 1 is retained on the activated platelet membrane following platelet activation. Haematologica. (2020) 105:2824-33. doi: 10.3324/haematol.2019.230367

100. Powers ME, Becker RE, Sailer A, Turner JR, Bubeck Wardenburg J. Synergistic action of Staphylococcus aureus alpha-toxin on platelets and myeloid lineage cells contributes to lethal sepsis. Cell Host Microbe. (2015) 17:775-87. doi: 10.1016/j.chom.2015.05.011

101. Navarro-Núñez L, Pollitt AY, Lowe K, Latif A, Nash GB, Watson SP. Platelet adhesion to podoplanin under flow is mediated by the receptor CLEC-2 and stabilised by Src/Syk-dependent platelet signalling. Thromb Haemost. (2015) 113:1109-20. doi: 10.1160/TH14-09-0762

102. Pircher J, Czermak T, Ehrlich A, Eberle C, Gaitzsch E, Margraf A, et al. Cathelicidins prime platelets to mediate arterial thrombosis and tissue inflammation. Nat Commun. (2018) 9:1523. doi: 10.1038/s41467-018-03925-2

103. Schulz C, VON BRÜHL ML, Barocke V, Cullen P, Mayer K, Okrojek R, et al. EMMPRIN (CD147/basigin) mediates platelet-monocyte interactions in vivo and augments monocyte recruitment to the vascular wall. J Thromb Haemost. (2011) 9:1007-019. doi: 10.1111/j.1538-7836.2011.04235.x

104. Abubaker AA, Vara D, Visconte C, Eggleston I, Torti M, Canobbio I, et al. Amyloid peptide $\beta 1-42$ induces integrin $\alpha \operatorname{IIb} \beta 3$ activation, platelet adhesion, 
and thrombus formation in a NADPH oxidase-dependent manner. Oxid Med Cell Longev. (2019) 2019:1-12. doi: 10.1155/2019/1050476

105. Bagge A, Schött U, Kander T. High-dose omega-3 fatty acids have no effect on platelet aggregation or coagulation measured with static and flow-based aggregation instruments and Sonoclot; an observational study in healthy volunteers. Scand $J$ Clin Lab Investig. (2018) 78:539-45. doi: 10.1080/00365513.2018.15 16477

106. Philipose S, Konya V, Sreckovic I, Marsche G, Lippe IT, Peskar BA, et al. The prostaglandin E2 receptor EP4 is expressed by human platelets and potently inhibits platelet aggregation and thrombus formation. Arterioscler Thromb Vasc Biol. (2010) 30:2416-2423. doi: 10.1161/ATVBAHA.110.2 16374

107. Pasterk L, Lemesch S, Leber B, Trieb M, Curcic S, Stadlbauer V, et al. Oxidized plasma albumin promotes platelet-endothelial crosstalk and endothelial tissue factor expression. Sci Rep. (2016) 6:1-11. doi: 10.1038/srep 22104

108. Gale BK, Jafek AR, Lambert CJ, Goenner BL, Moghimifam $\mathrm{H}$, Nze UC, et al. A review of current methods in microfluidic device fabrication and future commercialization prospects. Inventions. (2018) 3:60. doi: 10.3390/inventions30 30060
109. Bjornmalm M, Yan Y, Caruso F. Engineering and evaluating drug delivery particles in microfluidic devices. J Control Release. (2014) 190:139-49. doi: 10.1016/j.jconrel.2014.04.030

Conflict of Interest: The authors declare that the research was conducted in the absence of any commercial or financial relationships that could be construed as a potential conflict of interest.

Publisher's Note: All claims expressed in this article are solely those of the authors and do not necessarily represent those of their affiliated organizations, or those of the publisher, the editors and the reviewers. Any product that may be evaluated in this article, or claim that may be made by its manufacturer, is not guaranteed or endorsed by the publisher.

Copyright (c) 2021 Zhang, Ramasundara, Preketes-tardiani, Cheng, Lu and Ju. This is an open-access article distributed under the terms of the Creative Commons Attribution License (CC BY). The use, distribution or reproduction in other forums is permitted, provided the original author(s) and the copyright owner $(s)$ are credited and that the original publication in this journal is cited, in accordance with accepted academic practice. No use, distribution or reproduction is permitted which does not comply with these terms. 